ganz auberoldentlich langwierig, ermüdend, entstellend und versagen trotz aller aufgewandten Zeit und Mühen häufig noch obendrein. Wenn man daher über andere Mittel verfügt, Onychomykosen, und es gilt dies auch für die Psoriasis und das Ekzem der Nägel, sauberer, angenehmer und schneller de. finitiv zu heilen, so ist man auch verpflichtet, die alten Methoden zu verlassen und den Patienten nicht mehr mit diesen zu quälen, zumal eine sechs- bis neunmonatige Behandlung nach den alten Methoden oft ohne jeden Erfolg bleibt.

Ich war im Spätsommer genötjgt, mehrere Patienten an den Nägeln zu löntgenisieren. Die Kranken, deren zwei mir von außerhalb zugeschickt wurden, weil die medikamentöse Behandlung mit allen nur erdenklichen Mitteln versagt hatte, wollten nach so langer Leidenszeit bestimint von mir wissen, wie die Röntgenbestrahlung an den Nägeln verliefe, wie oft bestrahlt werden müsse, ob eine vollkommene Heilung eintrete, ob die Nägel abgingen, ob sie wieder nachwachsen, oder ob anstatt einer Heilung gar eine Verschlimmerung, ein Schaden zu befürchten sei; dem ersten der Patienten konnte ich nur das versprechen, daß ich ihm nicht schaden würde. Das Weitere würde sich zeigen. In der Literatur nämlich kann man sich über alle diese Fragen nicht belehren. So war ich also ganz auf inich selbst angewiesen, und ich verließ mich auf meine Erfahrungen, die ich mit den Röntgenstrahlen an der Haut kranker Menschen bisher gesammelt hatte. Es kam mir darauf an, sofort dje richtige Dosierung zu finden, welche in wenigen Sitzungen die volle Wirkung entfaltet. Ich glaube daher, eine Lücke

\section{Die Behandlung kranker Nägel mit Röntgenstrahlen.}

Von Dr. Carl Schindler, Spezialarzt fur Hautkrankheiteı in Berlin.

Im Heft 2, 88. Band des Archivs f. Dermat. u. Syphil. Januar 1908, erschien eine Arbeit aus der Abteilung für Hautund Geschlechtskrankheiten der städtischen Krankenanstalten Kölns von F. R. M. Berger: "Die Behandlung der Mikrosporie, der Trichophytie und des Favus mit Röntgenstrahlen“. In dieser sehr unterrichtenden Arbeit bemerkt der Verfasser und ebenso in seiner jüngsten Veröffentlichung $\mathrm{Kien}$ böck, daß die tatsächliche Spärlichkeit von Publikationen auf diesem Gebiete jede einschlägige Mitteilung rechtfertige, umsomehr, als kein Objekt für die Radiotherapie so dankbar sei als der Favus. Wenn nicht prompte Heilung eintritt, so liegt das an ungenügender Epilation durch schlechte Technik, wodurch vereinzelte Krankheitsherde zurückbleiben. Noch viel spärlicher aber ist die Literatur über die Behandlung von Pilzkrankheiten und andern Erkrankungen der Nägel mit Röntgenstrahlen. Ich glaube, wenn ich auch, des vergeblichen Suchens müde, durchaus nicht die ganze in- und ausländische Literatur durchforscht habe, dennoch behaupten zu dürfen, dab in den Lehrbüchern und in Einzelarbeiten so gut wie nichts darüber vorhanden ist. Insbesondere fehlt jede Mitteilung, mit welcher Technik kranke Nägel bestrahlt werden müssen, um eine Heilung derselben zu erzielen.

Es ist charakteristisch, daß Sabourau d in seinem vorzüglichen Handbuch der Dermatologie topographique 1905 bei der Besprechung der Behandlung der Pilzkrankheiten des behaarten Kopfes, der 'Trichomykosen, die Röntgenbestrahlung genan beschreibt, in die vorderste Reihe stellt und einen Unterschied maclit zwischen der Radiotherapie und den alten Methoden, (man kann wohl sagen, veralteten Methoden), deren Existenzberechtigung or nur noch darin sieht, daß nicht jeder über einen Röntgenapparat verfügt (und auch mit ihm zu arbeiten versteht). Um so bezeichnender ist es, da/3 dieser große Kenner der Trichomykosen in dem Kapitel über die Nagel für die Belıandlung der Onychomykosen die alten Methr.den empfiehlt und beschreibt, Pinselungen oder die chirurgische Entfernung der Nägel, ohne der Röntgenstrahlen auch nur mit einem Worte zu erwähnen.

Die alten Methoden, und man darf auch für die Onycho. mykosen sagen, veralteten Methoden bestehen in Pinselungen oder Entfernung der Nägel, sei es in Narkose durch einen chirurgischen Eingriff, sei es durch allmähliche Erweichung und Lockerung der Nägel durch Umschläge mit Kalilauge oder durch Kautschukverbände. Diese Methoden sind auszufüllen und manchem Alzte aus der Verlegenheit zu helfen, anderseits auch zu allgemeinerer Anwendung der Röntgenstrahien bei Nagelkrankheiten anzuregen, wenn ich bekannt gebe, mit welcher Technik ich in mehreren Fällen, und zwar stets mit derselben Technik überraschende Erfolge hatte.

\section{Onychomykosis.}

Im Spätsommer 1907 wurde mir ein Domänenpächter von außerhalb uberwiesen, der vermutlich durch eines seiner Tiere eine Pilzkrankheit erwarb, die fast zu gleicher Zeit alle zehn Fingernägel ergriffen hatte. Die Haut selbst war und blieb während der langen Dauer (ein Jahr) der Krankheit verschont. Nachdem durch alle möglichen parasitiziden Pinselungen, Salben, Umschläge nicht die mindeste Besserung erzielt worden war, sollte ich nun einen Versuch mit der Rontgenbestrahlung machen. Der Kollege, selbst ein erfahrener Dermatologe, schrieb mir dazu, dal3 sich der Verlauf nngefähr so gestaltet habe: „Zuerst war der vordere Rand des Nagelbettes ergriffen, von hier kroch die Affektion ganz allmählich unter die Nagelplatte nach hinten, letztere zum langsamen Absterben bringend. Zwischen Nagelplatte und Nagelbett etablierte sich eine bei den einzelnen Nageln verschiedene, mächtige, trockene, krümelige, mörtelartige Masse von graungrïner Farbe. Die Nagelplatte wurde trübe, rissig, lamellös geschilfert, am vorderen Rande dünn und scbarfkantig unter beständiger Verkürzung in der Richtung von vorn nach hinten. Eine sekundäre Entzündung des Nagelfalzes bestand nicht, die Affektion blieb stets trocken uud näßte nie.“

Als ich den Pächter sah, waren sämtliche Fingernägel um die Hälfte, die Ring- und kleinen Fingernägel sogar um ein Drittel verkürzt. Die Nagelplatten waren tiefschwarz-grünlich verfärbt. und undurchsichtig, einige zeigten stärkere Längsstreifung; locker waren die Nägel nicht, die Lunula war durch die gleichmälig tiefe Schwarzgrünfärbung auch nicht mchr andeutungsweise zu erkennen Das Nagelbett war zum Teil mit trockenen, krümeligen, grau-grünen Massen bedeckt am Rande der verkleinerten Nagelplatte und unter derselben. Die mikroskopische Untersuchung der grau grunen Massen und kleiner Stückchen der Nagelplatte nach 24 stündiger Erweichung der letzteren in $331 / \% \%$ Kalilauge ergab an einzelnen Stellen sicher als Sporen zu erkennende Gebilde und kurze Reste von Mycelien, aber doch schon so spärlich, daß ich ohne das klinische Bild und die Vorgeschichte mikroskopisch die Diagnose einer Onychomykese bestimmt zu stellen nicht gewagt hätte. Aber daß es sich um eine solche handelte, daran konnte auch wieder kaum gezweifelt werden. Wenn auch die Unterscheidung zwischen Favus und Trichophytie nur durch die Kultur gefällt werden kann, so wird man doch nach der Verfärbung und mangels jeglicher Scutnla eher zur Annahme einer durch Tiere auf den Menschen übertragenen Trichophytie als eines Favus sich hinneigen.

Dieser Zustand war dem Pächter sehr unangenehm, weil er in Gesellschaften nicht mehr zu Tisch gehen konnte. Eine Entzündung der umgebenden Haut bestand nicht, es fehlte jede entzlindliche Rötung. Ich konnte daher energisch bestrahlen. Ich wollte von 
vornherein ein kräftiges Erythem und dadurch Aufheliung und Heilung der Nägel oder eventuell durch noch stärkere Bestrahlung eine Abstoßung der Nägel erzielen. Ich bestrahlte am 23., 25, 27, 28., 30. September, 1. und 2. Oktober jeden Nagel 15 Minuten mit einer mittelweichen Hirschmannschen Monopolröhre in $12 \mathrm{~cm}$ Entfernung des Focus der Röhre ron der Haut, immer untcr denselben Stromverhältnissen. $7 \mathrm{~cm}$ Funkenstrecke eines $60 \mathrm{~cm}$ Indıktors, mit Hirschmannschem G]eitkontaktunterbrecher 2200 Unterbrchungen, 1,5 Ampère, und 0,5 Millianpère im sckundären Stromkreis. Ich weil aus meiner Erfahrung daß3 ich bei sieben Bestrahlungen unter diesen stets konstanten Stromverhältnissen in 8-10 Tagen ein kräftiges Erythem erziele.

Es wurden zuerst bestrahlt beide Daumennägel, dann die vier ibrigen Nägel je der rechten und der linken Hand, sodab die Bestrahlung aller zehn Nägel jedesmal $3 / 4$ Stunden duuerte. Am 12. Oktober, zehn 'Tage nach der letzten Bestrahlung, trat unter eben beginnender Aufhellung der Nägel ein Erythem der nicht abgedeckten Haut des Nagelwalles ein, welches sich bis in die dritte

Fig. 1

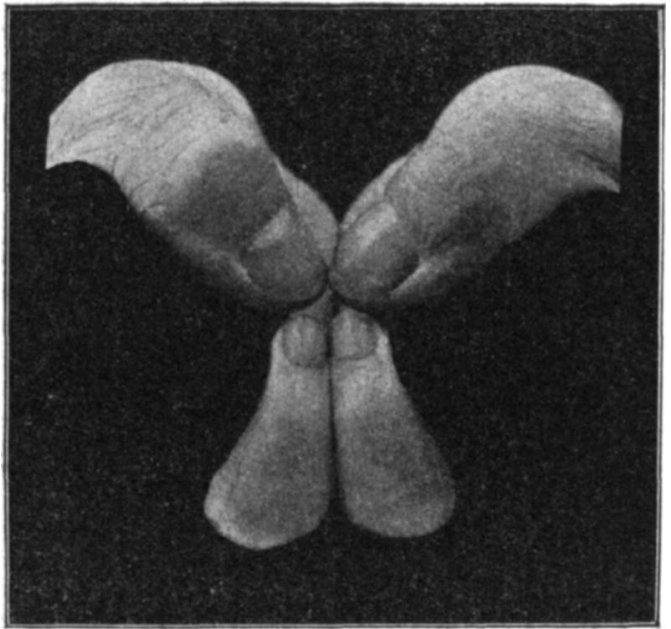

Gute Stellung, gleichmăßige Bestralılung.

Woche nach der letzten Bestrahlung verstärkte und konstant erhielt, um dann bis zum Ende der fünften Woche vollständig wieder zu verschwinden, Es traten weder Exkoriationen, Bläschen noch Pigmentierungen auf. Die Haut war nur gespannt, stark gerötet und brannte spontan etwas, wogegen ein Druck nicht schmerzhaft war. Einfettung nur mit Taseline genïgte, die subjektiven $\mathrm{Be}$ schwerden zu beseitigen.

In dieser Zeit nun bis zum Ende derfïnften Woche hatten sich sämtliche Nägel mehr und mehr aufgehellt. Die Da umennägel, die Nägel der Mittel- und Ringfinger waren vollkommen rosig durchscheinend geworden. Die bröckligen Aullagerungen des Nagelbettes waren ganz verschwunden. Nur die Näge der Zeige- und der kleinen Finger waren zwar aufgehellt aber zeigten doch noch die schwarzgrüne Verfärbung und die Auflagerungen.

Dieser Teilerfolg gerade bei diesen Nägeln erklärt sich aus Fig. 3 durch die ungleichmäßige Bestrahlung, indem gerade diese Finger nur Randstrahlen, die andern aber Focusstrahlen erhalten

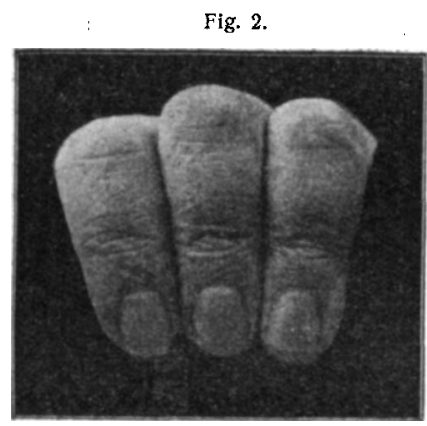

Gute Stellung. hatten. Ich empfehle daher nach weiteren Versuchen, wenn man alle zehn Nagel bestrahlen mul, Fig. 1 als Stellung 1 und dann Fig. 2 als Stellung 2 je für die rechte und linke Hand. In jeder Stellung wird $15 \mathrm{Mi}$ nuten bestrahlt, und man erhält gleichmäßige Reaktionen. A m 20. Norember 1907, zwe Monate nach der letzten Bestrahlung röntgenisierte ich, auf Wunsch des Patienten auch die ganz ges unden Nïgel noch einmal unter denselben Stromverhältnissen wie früher, jedoch nur viermal, täglich drei Viertelstunden. Ein Erythem trat jetzt nicht auf, und nach 14 Tagen waren nun alle Nägel schön rosig gefärbt und frei von Auflagerungen. Salben, Pinselungen wurden während der Röntgenknr nicht gebraucht. Die mikroskopisclıe Untersuchung eines

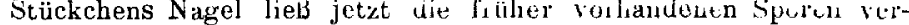
missen. Fs stellte sich aber auch sichtlich wieder das Wachstum aller N $\mathrm{N}$ gel ein wenn auch in ungleichmidigem Grade. Es war geradezu zanberhaft, wie nach 1 jähriger vergeblicher medikamentöser Behandlung in nicht ganz zwei Monaten nit nur sieben starken und vier schwäche ren Bestrahlıngen die tief schwarz-grïnen Nägel wieder rosige Farbe annahmen.

lch habe mir nun die Frage vorlegen müsselı, wodurch die Röntgenstrahlen in so über. rascliender Weise, wie
Fig. 3

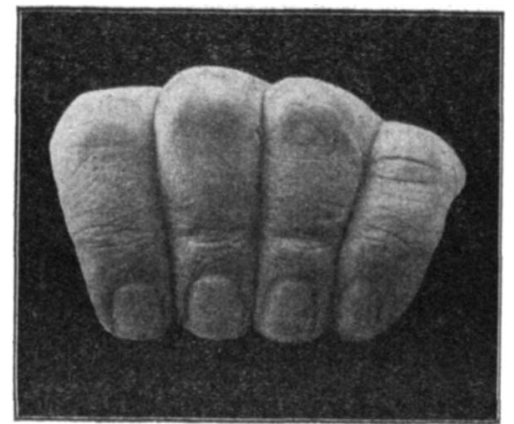

Schlechtere Stellung, ungleichmäßige Bestrahlung. durch einen geheimnisvolten Zauber, dicse bisher vergeblich behandelte Onychomykose zur Heilung brachten. Wir wissen, daß die Röntgenstrahlen nicht bakterizid wirken, allenfalls erst be so andauernden und starken Bestrahlungen, wio wir sie dem Menschen nicht angedeihen lassen können. Wenn sich auch hier die Meinungen noch gegenüberstehen, so sprechen doch manche Tatsachen gegen eine bakterizide Wirlung der Röntgenstrahlen. Krause und Jastram fanden bei Versuchs anordnungen, die dell in der Praxis angewandten Röntgenbestrahlungen möglichst entsprcchen, jndem die Kulturen, ähnlich wie die hranken Menschen, täglich nur beschränkte Zeit (z. B. drei Wochen tïgrich 20 Minuten) bestrahlt wurden, daB in keinenı Fall und bei keiner Bakterienart die Röntgenstrahlen die Bakterien getötet oder ihr Wachstum etwa nur gehemmt hatten. Wir wissen rom lavus des Kopfes, dak durch die epilierende Röntgendosis die Pilze niclıt zerstört werden, denn Kulturen von den epilierten, Pilze enthaltenden Haaren singen mit unveränderter Kraft an. Da die Nägel aber nicht abgestoßen und auf diese Weise die Pilze mit ihnen entfernt wur den, so mub man auch hier die Einwirkung der Röntgenstrahlen auf Bakterien, Pilze, im menschlichen Gewebe, als eine indirekte, als eine den Nührboden durch die Schädigung der Gefäße und der Gewebszellen verschlechternde betrachten. Wenn auch der Favus des Kopfes mit einer sehr viel schwächeren Dosis bestrahlt wird, sodah ohne Erytlem gerade die Haare ausgehen, und zur Heilung der Nägel eine sehr kräftige Erythemdosis erforderlich war, so möchte ich trotzdem nicht behaupten, daß durch diese die Pilze direkt getötet wurden. In frischeren Fällen, die für einen Kulturerfolg sicherer sind, kann man ja durch Kulturen kleinster Nagelstückchen vor und nach der Bestrahlung bis zu einem starken Erythen die bakterizide Kraft dieser die Krankheit selbst heilenden Röntgendosis feststellen. Ueber die Einwirkung der Röntgenstrahlen auf tierisches Gewebe haben Krause und Ziegler durch experimentelle Untersuchungen sich dahin geäußert, daß in ausgesuchter Weise gerade diejenigen Organe geschädigt werden, welche durch äußerst lebhafte Zellproduktion und Zellansatz ausgezeichnet sind, wie es z. B. bei den Haut- und Haarfollikeln der Fall ist und, wie ich wohl hinzufügen darf, auch bei den Zellen der Nagelmatrix. Als notwendiges, anatomisches Substrat für eine wirksame Röntgentherapie betrachten Krause und Ziegler junge, leicht zu beeinflussende Zellen. Da die Röntgenstrahlen auf die Bakterien selbst nicht einwirken, was auch Sabouraud, Kienböck bestätigen, so bleibt nur die Schädigung des Zellulargewebes als Verschlechterung des Nährbodens zur Erklärung für die parasitizide klinische Wirkıng der Röntgenstrahlen übrig. Bordier führt die Verdün. nung der Nagelplatten bei dauernder Bestrahlung auf Ernährungsstörungen der Zellen der Nagelwurzel zurück. Bordier meint, daß die Röntgenstrahlen Eiweiß in colloidalen Zustand überführen, sodaß es nicht mehr assimiliert werden kann. ${ }^{1}$ )

1) Während der Korrektur dieser Arbeit sah ich am 31. Januar cr. den Patienten noch einmal wieder. Die Nägel haben ihre normale Färbung und sind bis an die Fingerkuppen normal gewachsen, Einzelne Nägel beginnen jetzt sich eben abpünormal gewachsen, der Basis der Lunula einen ganz feinen, horizontalen Spalt bemerkt. unter dem der neue Nagel bereits sichtbar ist. 
Es entzieht sich meiner Kenntnis, ob und mit welchem Erfolge Haustiere, Katzen, Hunde, speziell wertvolle Jagdhunde, im Beginn der Erkrankung an Räude bestrahlt wurden. Diese Bestrahlung im Beginn der Erkrankung dürfte um so aussichtsvoller sein, als sich ja zuerst, ganz isoliert, kleinere, wie bestäubt aussehende Herde bilden. Hancher Jäger wird durch rechtzeitige Bestrahiung seinen wertvollen Hund vor dem Erschieben bewahren.

\section{Psoriasis und Ekzem.}

Zufälligerweise hatte ich im November 1907, als ich die onychomykosis gerade zum zweiten Male bestrahlte, Gelegenheit, noch zwei Fälle von Psoriasis der Nägel und später ein Ekzem derselben mit Röntgenstrahlen zu behandeln.

In dem einen Fall von Nagelpsoriasis handelte es sich 1 Im eine junge Dame, die nach langer, vergeblicher Behandlung mit Pinselungen mir zur Bestrahlung von einem auswärtigen Kollegen uberwiesen worden war. Die junge Dame hatte niemals an irgendeiner anderen Stelle der Haut, sondern stets nur an den Nägeln beider Hände Psoriasiseffloreszenzen. Die Rezidive waren des öfteren aufgetreten. Man sah an den Nägreln die charakteristischen kleinen, kaum stecknadelkopfgroßen Grïbchen, aber außerdem bestanden weißliche Verfärbungen der Nagelplatte. Andere pathologische Veränderungen der Struktur und Gestalt der Nägel, sowie der Nagelwälle bestanden nicht. Ergriffen waren Zeige-, Mittel- und Ringfinger der rechten $\mathrm{Hand}$, Zeigefinger und Mittelfinger der linken Hand. Da ich Erfahrungen über die Bestrahlung psoriatischer Nägel nicht liatte und es sich zumal um eine Dame handelte, wollte ich kein Frythem durch die Bestrahlung erzeugen, indem ich mich an die Vorschrift und an die Erfahrungen hielt, die mir durch die Bestrahlung psoriatischer Haut zur Verfügung standen. Es ist bekannt, da $B$ die psoriatische Haut schon auf leichte Bestrahlungen reagiert und daß zu starke Bestrahlungen, welche ein Erythem erzeugen, als traumatischer Reiz auf die Psoriasis wirken und deren weitere, oft rapide Ausbreitung zur Folge haben können.

Ich bestrahlte daher in derselben Versuchsanordnung, die ich schon geschildert habe, die Nägel dreimal; zweimal, je einmal an zwei aufeinanderfolgenden Tagen, das drittemal lag der Sonntag, an dem nicht bestrahlt wurde, dazwischen. Nach 14 Tagen war noch keine Veränderung aufgetreten, ein Erythem war nicht erfolgt, aber auch keine Besserung. Ich wollte nun noch einmal bestrahlen, und zwar jetzt mit einer Erythemdosis. Leider schrieb die Patientin ab, sie gehe auf Reisen und wolle vorläufig die Behandlung unterbrechen.

Ich war daher sebr erfreut, kurz darauf bei einem Mann mit allgemeiner Psoriasis noch einmal psoriatische Plaques der Nägel bestrahlen zu könnelı. Auch hier bestanden sonst keine weiteren Veränderungen. Ich benutzte daher die inzwischen gewonnene Erfahrung, daf eine Epilationsdosis wirkungslos ist, und ich bestrablte gleich die Nägel mit derselben Erythemdosis wie die Onychomykosis, sieben Tage. Ergriffen waren rechts Zeige- und Ringfinger, links Mittel- und Ringfinger. Und dieses Mal hatte ich wieder einen Erfolg, ich bekam wieder, und zwar schon am neunten Tage, ein beginnendes, kräftiges Erythem, das sich in den nächsten 14 Tagen bis in die dritte Woche steigerte, dann aber wieder bis zur Mitte der fünften Woche sich vollständig verloren hatte. In dieser Zeit verkleinerten sich die weißen Plaques mehr und mehr, indem an ihrer Stelle die Nagelplatte wieder rosig durchschien und schließlich von den Plaques nichts mehr zu sehen war. Zur Technik möchte ich hier noch nachtragen, da $B$ ich kleine Hiilsen von Bleiblech über die Nagelglieder der Finger stülpe und einen entsprechend großen Ausschnitt für die Nägel und die Haut des Nagelwalls mache. Die Phalangen der Finger und der Hand werden außerdem in ublicher Weise abgedeckt.

lch konnte schließlich noch ein trockenes Nagelplattenekzem bei einem Kupferstecher bestrahlen.

Die Haut des linken Mittel- und Zeigefingers war ebenfalls ekzematös erkrunkt, infiltriert, schuppend, trocken und mäßig juckend. Die Nagelplatten waren gelb-bräunlich verfärbt, glanzlos, gefurcht. Unter guter Abdeckung der Haut, nur eben die Nagelfalze blieben frei, bestrahlte ich die Nagelplatte wieder erfolgreich mit dor vollen Erythemdosis. Ich möchte an diesen Fall den von Pfahler anschließen, der eine chronische Staphylococcenparony.chie, die in 20 Jahren fünfmal rezidiviert war, wobei jedes Rezidiv ein Jahr lang bis zur Heilung brauchte, in drei Monaten mit Röntgenbestrahlung vollkommen ausheilte.

lch bin am Schlub. Wenn meine Erfahrungen bei der Seltenheit der Nagelerkrankungen für den einzelnen Arzt auch noch gering sind, so sind sie doch so ermutigend und überraschend, daß man auch fül die Nagelerkrankungen, deren medikamentöse Behandlung für den Patienten wie für den Arzt ein Kreuz ist, die Anwendung der Röntgenstrahlen als Heilmittel gegenüber den alten Methoden als die normale Therapie bezeichnen mul, sichel für die Onychomykosen, Psoriasis und gewisse Forinen der Ekzene und Paronychien. Die Behandlung mil Röntgenstlahlen ist schnellwirkend, sauber, bequem, schmerzlos und ebenso angenehm für den Arzt und den Patienten, wie die alten Methoden unangenehm und entmutigend sind. Gern bin ich bereit, 'zur Verarbeitung einer kasuistischen Statistik die Mitteilung von Kollegen entgegenzunehmen, die Nagelerkrankungen zu bestrahlen Gelegenheit hatten. Der Einzelne wird immer nur wenige Fälle sehen, wer weiß, wann ich selbst wieder einmal gleich mehrero Nagelerkrankungen werde bestrahlen können. Wie arm die Literatur ist, hat sich ergeben. Es müssen daher die einzelnen Fälle gesammelt werden, und vor allem müssen die Röntgenstrahlen viel häufiger als bisher zur Heilung von Nagelkrankheiten verwendet werden. Der Kranke hat heute ein Recht auf die schnellere Heilung durch die Bestrahlung.

Resümee. Die Röntgenbestrahlung heilt Mykosen, Psoriasis, Ekzeme der Nägel in kurzer Zeit. Man muß so kräftig bestrahlen, dab ein stälkeres Erythem auftritt. 